\title{
RETRACTED ARTICLE: Improving the vocational education based Japanese teaching research approach using deep learning techniques
}

\author{
Shuo Tian ${ }^{1}$ \\ Published online: 15 June 2020 \\ (C) Springer Science+Business Media, LLC, part of Springer Nature 2020
}

The Editor-in-Chief has retracted this article. The article was accepted as part of a guest-edited special issue in Current Psychology. Before the special issue was finalized, the Editor-in-Chief detected problems with editorial handling and peer review and decided not to proceed with the special issue. Post publication peer review found that this article is out of scope for the journal and does not meet the standards required by the journal. The author has not responded to any correspondence from the Editor about this retraction.

The online version of this article contains the full text of the retracted article as Supplementary Information.

Supplementary Information The online version of this article (https://doi. org/10.1007/s12144-020-00833-x) contains supplementary material, which is available to authorized users.

Shuo Tian

tianshuo0451@163.com

1 School of Foreign Languages, Harbin University of Science and Technology, Harbin 150080, China 